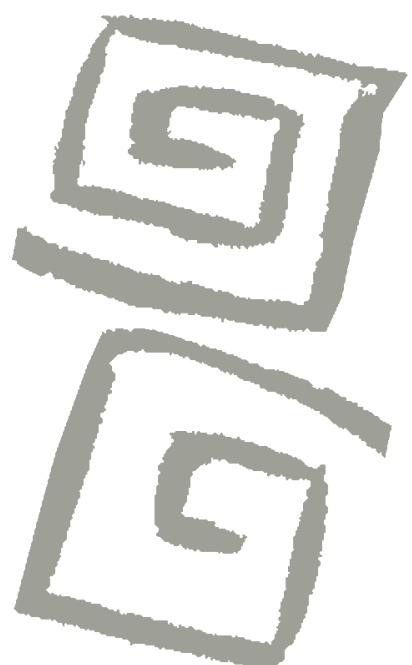

\title{
Cuidados informales de larga duración en España: retos, miradas y soluciones
}

\section{Long-term informal care in Spain: challenges, views and solutions}

${ }^{1}$ Doctora en Antropología Médica. Investigadora colaboradora, Medical Anthropology Research Centre, Universitat Rovira i Virgili, Tarragona, España. $\bowtie$ iD
RESUMEN El cuidado de la enfermedad crónica a todas las edades representa un problema social y de salud pública en aumento. En España, debido a la insuficiente cobertura estatal, cerca del $80 \%$ de los cuidados de larga duración los realiza la red social del enfermo. A partir de una investigación etnográfica sobre cronicidad, desarrollada entre 2009 y 2013 en el estado español, este artículo analiza la problemática de los cuidados informales de larga duración sobre la base de las narrativas de adultos de mediana edad con enfermedades crónicas. Los resultados señalan los factores que condicionan, favorecen o limitan el cuidado informal: la situación personal, doméstica y familiar del enfermo; el curso cíclico de la enfermedad crónica; y la respuesta social a la enfermedad. Las limitaciones del modelo de cuidado informal emplazan a escuchar las voces de los afectados para conocer sus necesidades reales y adecuar los planes y programas de atención a la cronicidad.

PALABRAS CLAVES Enfermedad Crónica; Autocuidado; Antropología Médica; Políticas Públicas; España.

\begin{abstract}
Caring for chronic illnesses at all ages represents a growing social and public health problem. Due to insufficient public coverage in Spain, around $80 \%$ of long-term care is provided by the sick person's social network. Based in ethnographic research on chronicity carried out between 2009 and 2013 in Spain, this article analyzes the problem of long-term informal care for middle-aged adults with chronic diseases. The results highlight the factors that condition, enhance or limit informal care: the personal, domestic and family situation of the sick person; the cyclical course of chronic diseases; and the social response to illness. The limitations of the informal care model suggest the need to listen to the voices of those affected in order to understand their real needs and adapt official programs oriented towards chronicity accordingly.
\end{abstract}

KEY WORDS Chronic Disease; Self Care; Medical Anthropology; Public Policies; Spain. 


\section{INTRODUCCIÓN}

El incesante aumento de personas que padecen alguna enfermedad crónica representa un problema social y de salud pública para los países europeos con Estado de bienestar, como España. Según la Organización Mundial de la Salud (OMS), actualmente las enfermedades crónicas suponen más de la mitad de la carga mundial de morbilidad, y es la causa del $75 \%$ de las muertes anuales, por lo que se considera, incluso, una nueva epidemia ${ }^{(1,2)}$. Según la última Encuesta Europea de Salud en España (EESE) de 2014 ${ }^{(3)}$ el $59,83 \%$ de la población mayor de 16 años padece al menos un proceso crónico, un aumento significativo respecto del $45,6 \%$ reportado en la encuesta anterior de 2009. Además, algunas personas sufren más de una enfermedad crónica, lo que se conoce como comorbilidad: en España, el 22\% de la población padece dos procesos crónicos o más, y dichos porcentajes tienden a incrementarse con la edad ${ }^{(4)}$. En Cataluña -una de las 17 Comunidades autónomas de España en las que se desarrolló esta investigación- tres de cada cuatro adultos declara tener una enfermedad crónica que, a su vez, es la causa principal de discapacidad y/o dependencia derivada (hasta un $6 \%$ de la población $)^{(5)}$. Las enfermedades crónicas generan el $75 \%$ del gasto sanitario y el $80 \%$ del gasto farmacéutico para los países occidentales ${ }^{(6)}$, y se estima que el $80 \%$ de las visitas al médico de atención primaria se deben a algún padecimiento crónico ${ }^{(4,6)}$. Las cifras se revelan altas, pero ¿de qué nos están hablando estas cifras?

Estas cifras ponen de relieve la preocupante carga de morbilidad y mortalidad y su impacto en una sociedad que envejece con muchos enfermos crónicos. Ello supone serias implicaciones económicas para los países europeos con Estado de bienestar y un reto para la provisión de servicios sanitarios y sociales de acuerdo con los principios de universalidad e igualdad de acceso para todos los ciudadanos. En el caso español, el reto se acrecienta en un contexto de crisis económica, de recortes presupuestarios y de la necesidad de contener el gasto. En este contexto tan poco prometedor ¿qué pasa con los enfermos crónicos que necesitan y necesitarán asistencia continuada de por vida?

Las enfermedades crónicas generan necesidades crónicas de atención y, por tanto, requieren de cuidados a largo plazo: el conocido long term care (LTC). Es importante subrayar que todas las enfermedades crónicas requieren cuidados en mayor o menor grado y conllevan rutinas de autocuidado para el día-a-día del enfermo crónico. Este grupo de enfermedades precisan de un modelo de atención diferente al que durante décadas ha funcionado para tratar las enfermedades agudas. La principal diferencia radica en cuidar frente a curar, puesto que las enfermedades crónicas son incurables, hasta que la medicina no demuestre lo contrario. Por ello, lo único que se puede hacer por el momento es cuidar/atender las enfermedades crónicas, es decir, cuidar/atender a los enfermos crónicos, ayudándolos también a que ellos cuiden de su salud. Pero ¿cómo se hace y quién se ocupa de ello?

La problemática de la responsabilidad de la atención y el cuidado de las personas que padecen enfermedades crónicas tiene una doble dimensión: pública y privada. Por un lado, el Estado de bienestar provee servicios sanitarios y sociales a los ciudadanos con enfermedades crónicas a través de programas y planes de actuación, lo que conocemos por políticas públicas. Por otro lado, encontramos a la persona enferma y su red social cercana de apoyo, que pondrán en marcha estrategias para atender el día-a-día de las necesidades, a través de lo que conocemos por autoatención y cuidados informales. ¿Cuál es el equilibrio entre lo público y lo privado en el caso español?, ¿qué repercusiones tiene para el individuo enfermo y su familia o su entorno cercano?

En este artículo se dará cuenta del fenómeno de los cuidados informales de larga duración en España para adultos con enfermedades crónicas desde la perspectiva de la antropología médica, a partir del análisis de las narrativas de la enfermedad de los participantes de la única investigación etnográfica 
sobre cronicidad desarrollada recientemente en el estado español ${ }^{(7)}$. El objetivo es mostrar las limitaciones del modelo del cuidado informal, emplazando a revisar los discursos y prácticas con relación a los procesos de salud, enfermedad, atención y cuidado (PSEAC) de larga duración.

\section{METODOLOGÍA}

Este artículo se basa en parte del material etnográfico recogido durante $\mathrm{mi}$ investigación doctoral en antropología médica sobre la experiencia y la gestión de la cronicidad en adultos, que desarrollé en Cataluña (España) entre 2009 y $2013^{(7)}$. El objetivo de la investigación era profundizar en la experiencia y la gestión del día-a-día de la cronicidad, entendida como una categoría analítica que incluye diferentes tipos de enfermedades o malestares, biomédicamente diagnosticados o no, que persisten durante un largo período de tiempo.

Para ello, se seleccionó un grupo heterogéneo de participantes con diversas afectaciones crónicas físicas, también denominadas somáticas u orgánicas. Se trató de obtener una muestra diversa de diferentes padeceres crónicos con afectaciones leves o moderadas para la vida diaria a partir de los diversos campos de especialización médicos: enfermedades cardiológicas, respiratorias, endocrinas, digestivas, musculoesqueléticas, etc. La muestra final proporcionó participantes con diferentes enfermedades o padeceres crónicos tales como celiaquía, diabetes, enfermedades de la tiroides, hipertensión, cardiopatías, Enfermedad Pulmonar Obstructiva Crónica (EPOC), asma, bronquitis crónica, síndrome de fatiga crónica, fibromialgia, osteoporosis, artritis, procesos cancerígenos, enfermedades degenerativas en estado inicial, e incluyó participantes con malestares crónicos todavía sin diagnóstico biomédico preciso.

Se escogieron participantes entre 30 y 50 años, grupo etario que se acostumbra a denominar adultos o adultos jóvenes. El grupo de edad concreto fue escogido por tres razones:
1) metodológicas, la necesidad de acotar una muestra esencialmente heterogénea y amplia dentro de una temática amplia; 2) científicas, al tratarse de un grupo infraestudiado y a menudo invisibilizado en la literatura sobre cronicidad, y 3) impacto de una enfermedad crónica, para dar respuesta al principal objetivo de conocer el impacto en una edad en la que se (pre)supone que uno debe estar sano, socialmente activo y laboralmente productivo.

Los participantes se seleccionaron de diversas maneras: por el método de bola de nieve, a partir de un centro de atención primaria, el hospital de referencia de la zona geográfica en la que se desarrolló la investigación, y a partir de asociaciones de pacientes y de contactos de la investigadora en jornadas o charlas públicas relacionadas con la temática. El trabajo etnográfico de las narrativas de la enfermedad se basó, fundamentalmente, en los relatos de las personas en las entrevistas en profundidad, $(n=20)$, las observaciones realizadas en un grupo de ayuda mutua de una asociación de personas afectadas de fibromialgia y síndrome de fatiga crónica $(n=12)$, y en un grupo terapéutico de un hospital para pacientes de la unidad de dolor crónico $(n=6)$. La etnografía se tejió incluyendo las narrativas de algunos diarios personales de enfermos $(n=4)$, libros autoetnográficos $(n=11)$, narrativas disponibles en blogs, y sitios web de asociaciones de diversas enfermedades $(n=6)$ e información proveniente de lo que algunos autores denominamos etnografía informal y que son narrativas que aportan datos complementarios a la muestra principal objeto de la investigación.

Antes de cada entrevista, se revisó y firmó por duplicado el documento de consentimiento informado; la investigadora guardó una copia y el participante otra. Para las observaciones no se requirió documento de consentimiento informado en ninguno de los grupos. Se obtuvo la aprobación para la investigación por parte del equipo directivo de la asociación de pacientes, y por parte del director de la Unidad de Dolor Crónico y del director médico del hospital donde se realizaron las observaciones. En todos los 
casos se siguió el Código de Buenas Prácticas en Investigación de la Universitat Rovira i Virgili, en donde se adscribió la investigación doctoral.

Los datos personales se trataron de acuerdo con lo que dispone la Ley Orgánica 15/1999, de 13 de diciembre, de Protección de Datos de Carácter Personal. Se atribuyó un pseudónimo a cada participante a excepción de aquellos que pidieron aparecer con su nombre real, y se procuró anonimizar los datos necesarios para preservar la confidencialidad de los participantes, sus allegados, centros de salud y/o profesionales sanitarios referenciados.

Las entrevistas y observaciones son el grueso etnográfico que informa este artículo parcial derivado de esta investigación doctoral. Los datos empíricos se discuten y se cruzan con la bibliografía existente desde la antropología y la sociología de la medicina sobre la experiencia de la enfermedad crónica y el cuidado informal. También se refieren los informes y publicaciones principales de los organismos públicos pertinentes, en este caso, los planes y programas de intervención para la atención a la cronicidad y a la dependencia.

\section{Marco conceptual y contextual}

Antes de adentrarnos en el análisis es preciso definir los conceptos y nociones que se utilizarán en este trabajo con relación a: 1) los sujetos de análisis y 2) los cuidados informales.

\section{Cronicidad, enfermos crónicos y grado de dependencia}

Utilizo el concepto de cronicidad entendida como una categoría analítica que incluye diferentes tipos de enfermedades o malestares, biomédicamente diagnosticados o no, que persisten durante un largo período de tiempo ${ }^{(7)}$ (a partir de 12 meses). En este sentido, aunque utilice los términos enfermedades crónicas, enfermos crónicos, enfermos o crónicos, como términos generales para referirme a los sujetos de estudio, cabe apuntar que algunas personas con problemas de salud crónicos no se definen como enfermos, ni se sienten enfermos (aunque realicen tareas de autocuidado diarias), y que no todas las personas con malestares o problemas de salud crónicos referirán que tienen una enfermedad ni que son enfermos crónicos, algunos ni tendrán un diagnóstico preciso. En este trabajo no discerniré sobre las diferentes percepciones de la experiencia personal del padecer crónico, que he explorado en trabajos anteriores ${ }^{(7)}$, pero por razones de finalidad analítica se hace necesario adscribirlos a un grupo genérico que denominaré (provisionalmente) enfermos crónicos.

Cabe especificar que para esta investigación se escogieron personas con enfermedades crónicas con limitaciones leves o moderadas para su vida diaria. ¿Quién define qué es leve o moderado? Más allá de lo que el sentido común dicte, según el contexto sociocultural, las valoraciones y percepciones sobre el grado de afectación variarán de unos individuos a otros, incluso entre estos y sus familiares o allegados, y con los profesionales que los atiendan. Entendiendo que no hay una única escala universal que valore el grado de afectación, se hace necesario escoger una para definir y acotar los términos. En el caso español, los grados leve y moderado se corresponden a los grados moderado y severo descritos por los profesionales, según el baremo de valoración de la dependencia (BVD) vigente en el estado español a raíz de la creación en 2004, y de la implementación desde 2006 hasta 2016 de la Ley 39/2006 conocida como Ley de Dependencia ${ }^{(8)}$ :

Grado I. Dependencia moderada: cuando la persona necesita ayuda para realizar varias actividades básicas de la vida diaria, al menos una vez al día o tiene necesidades de apoyo intermitente o limitado para su autonomía personal.

Grado II. Dependencia severa: cuando la persona necesita ayuda para realizar varias actividades básicas de la vida diaria dos o tres veces al día, pero no requiere 
el apoyo permanente de un cuidador o tiene necesidades de apoyo extenso para su autonomía personal.

Grado III. Gran dependencia: cuando la persona necesita ayuda para realizar varias actividades básicas de la vida diaria varias veces al día y, por su pérdida total de autonomía física, mental, intelectual o sensorial, necesita el apoyo indispensable y continuo de otra persona o tiene necesidades de apoyo generalizado para su autonomía personal.

Este tercer y último grado de "gran dependencia" no se contempla en este estudio, puesto que era un criterio de exclusión para la selección de los participantes. Además, configura una situación radicalmente distinta a los grados inferiores, en lo que respecta a los cuidados informales.

¿Esto quiere decir que la mayoría de los enfermos crónicos son dependientes? No, tal como lo perciben los propios afectados y tal como lo percibe la ley. ¿Tienen todos los enfermos crónicos su grado de dependencia reconocido oficialmente, tal como estipula la ley? Tampoco, se discrimina a un notable grupo de enfermos crónicos debido a los excluyentes criterios biomédicos de valoración de la dependencia que no tienen en cuenta la totalidad de las necesidades que los diversos enfermos crónicos refieren o presentan, por lo que no pueden beneficiarse de las prestaciones que la ley dispone. De ahí que quien acaba asumiendo el cuidado sea la persona enferma y su entorno cercano, a través de lo que conocemos como cuidado informal.

\section{Cuidados informales y autoatención: cifras $y$ definiciones}

Si bien hay cierta variabilidad en las cifras según los diferentes autores consultados, en España, entre el $75 \%$ y el $88 \%$ de los cuidados crónicos lo prestan las redes sociales de apoyo, fundamentalmente la familia más cercana, rol que continúa recayendo casi en forma exclusiva en las mujeres ${ }^{(9,10,11)}$. La mayor parte del cuidado recae en el ámbito privado, que denominamos apoyo informal o cuidado informal. Debo apuntar que, a lo largo del trabajo, se utiliza el término cuidado informal, ampliamente utilizado y consensuado en la comunidad científica, para facilitar el entendimiento de aquello a lo que nos estamos refiriendo, aunque con ciertas reticencias. Dicho término se tomará como categoría paraguas, la cual incluye otros términos similares como cuidados familiares, cuidados no profesionales o cuidados legos o profanos ${ }^{(12)}$. Estos cuidados, en general, se prestan de forma continuada y los realizan, principalmente, los familiares y personas cercanas al enfermo crónico. A pesar del uso extendido del término, también en las ciencias sociales, este proviene de una mirada profesional, biomédica y hegemónica del cuidado al enfermo.

Es un hecho paradójico denominar informal a las prácticas de cuidado y a los cuidadores principales que asumen dicho cuidado de un $75 \%$ a un $88 \%$ de los casos, algunos de forma permanente, cada día, durante semanas, meses y años. Esto de ninguna manera puede considerarse informal. ¿No estaremos subestimando y descalificando estas prácticas al considerarlas informales?, ¿no estaremos infravalorando los saberes expertos de los enfermos y su red social de apoyo? Evidentemente, la discusión sobre este término radica en el conocido conflicto de poder entre los saberes expertos de los profesionales de la salud y los saberes expertos (llamados profanos) de los enfermos y sus cuidadores, invisibilizados durante décadas y revalorados a la luz de las limitaciones de la cobertura sociosanitaria oficial. Sin embargo, la idiosincrasia y características de este tipo de prácticas que se llevan en el ámbito privado del domicilio (en casa) hace difícil conceptualizarlas como "formales". Coincido con Comelles ${ }^{(13)}$ cuando señala que:

Las características de este proceso de construcción de conocimientos: es decir una combinación de saberes empíricos, de habilidades, y de capacidad de manejar recursos muy dispares hace que me cueste hablar de los saberes populares 
(de eso se trata, de saberes populares), como de un dispositivo formal, pero a la vez es también impropio hablar, como hace la literatura sanitaria y a veces la sociosanitaria, de informal o de profano. $\mathrm{Ni}$ es informal, ni es profano, sino todo lo contrario. ${ }^{(13)}$

Por otro lado, se toma aquí el concepto de autoatención de Menéndez ${ }^{(14)}$, como marco de referencia en el que se llevan a cabo dichas prácticas de los cuidados y gestión de la cronicidad en el ámbito familiar y social, y como categoría analítica paraguas que incluye otros conceptos similares, como el autocuidado y la autogestión. Tal como lo define el propio autor, autoatención se refiere a:

\footnotetext{
...representaciones y prácticas que la población utiliza a nivel de sujeto y grupo social para diagnosticar, explicar, atender, controlar, aliviar, aguantar, curar, solucionar o prevenir los procesos que afectan su salud en términos reales o imaginarios, sin la intervención central, directa e intencional de curadores profesionales, aun cuando estos pueden ser la referencia de la actividad de autoatención. ${ }^{(14)}$
}

Entonces, ¿cómo se llevan a cabo estos cuidados informales o prácticas de autoatención en los enfermos crónicos adultos en España?

\section{EL RETO DE LOS CUIDADOS INFORMALES DE LARGA DURACIÓN EN LA EDAD ADULTA}

Sabemos que las enfermedades crónicas afectan al conjunto de la población, a todas las edades, si bien de forma predominante en adultos y ancianos. No obstante, no es lo mismo cuando el enfermo es un niño, un joven, un adulto joven, un adulto o un anciano, sobre todo, en lo que respecta a los cuidados informales. Las expectativas sociales y culturales -patrones e idealesque se tienen sobre una persona sana o enferma a diferentes edades tienen matices que inciden significativamente en las relaciones interpersonales entre unos y otros, y en la respuesta social ante la enfermedad ${ }^{(15,16,17)}$. En el contexto sociocultural occidental en el que se inscribe esta investigación, presuponemos que si un niño o joven está enfermo, sus progenitores (principalmente) y otros miembros de la familia se ocuparán de él y lo cuidarán. Similarmente, si una persona anciana está enferma, entenderemos que sus descendientes y/u otros familiares se harán cargo de cuidarla, y cuando esto no sea posible, el Estado proporcionará el cuidado a través de centros de día o residencias de ancianos. Pero ¿qué pasa en el grupo etario del medio?, ¿qué pasa cuando el enfermo tiene algo más que veinte, treinta o cuarenta años?, ¿quién se ocupa de ello?

Los resultados de esta investigación etnográfica mostraron las principales problemáticas de los cuidados informales al enfermo crónico a estas edades centrales de la vida y que tienen que ver, principalmente, con: 1) la situación personal, doméstica y familiar del enfermo; 2) el curso cíclico de la enfermedad crónica; y 3) la respuesta social a la enfermedad.

\section{Situación personal, doméstica y familiar}

Cuando se precisan cuidados de larga duración, la situación personal, doméstica y familiar del individuo enfermo es de vital importancia. Vivir solo, en pareja o en familia, el número de personas de la unidad doméstica, los roles en el seno de la familia, y las responsabilidades dentro y fuera de la casa son determinantes. Ello configurará diferentes redes de apoyo informal y determinará la posible respuesta informal a las diversas necesidades que presente el enfermo crónico. El perfil del enfermo crónico adulto joven muestra cuatro variantes principales: los que viven solos (singles), los que viven en pareja, los que viven en familia (pareja e hijos) y los que viven con sus progenitores. Cada grupo, 
presenta sus problemáticas y limitaciones en lo que al cuidado informal se refiere. Cabe apuntar que esta investigación no puede dar cuenta del papel de los hermanos y las hermanas de los participantes en lo que al apoyo y cuidado se refiere. Esto no quiere decir que dicha ayuda fraternal no se dé, sino que no ha sido encontrada en esta investigación. Tampoco se puede dar cuenta de personas con enfermedades crónicas que convivan o compartan vivienda con amigos que les presten apoyo o cuiden de ellos, puesto que dicha casuística tampoco ha sido encontrada.

\section{Los singles}

Nos encontramos en un momento sociodemográfico en el que, en comparación con un pasado no tan lejano, hay un mayor número de personas que viven solas, los denominados singles (adaptado anglicismo). La primera obviedad cae por su propio peso: los singles enfermos no tienen a nadie bajo el mismo techo que pueda cuidar de ellos. Dependiendo de las limitaciones que la enfermedad imponga, de la ayuda recibida en casa (del Estado o red social) y/o de la capacidad económica de contratar servicios -por ejemplo, para realizar las tareas de la casa-, la opción individual de vivir solo puede ser o no posible. Víctor ha podido "montarse la vida" de forma independiente:

Yo prefiero vivir solo. Mi madre viene $y$ me ayuda sobre todo a pasar el aspirador y otras cosas que yo no puedo hacer bien, pero hasta que pueda intentaré vivir solo. Es mejor. Yo hago mi vida y no molesto a nadie, y nadie me molesta.

(Víctor, 31 años)

Pero Víctor es la excepción en esta investigación. Es el único participante que (¿todavía?) se puede permitir (¿el lujo de?) vivir solo. Otros no pueden vivir solos, bien sea por problemas económicos, logísticos o por el tipo de padecimiento que tienen. Querer y poder vivir solo a pesar de la enfermedad responde a la necesidad de preservar una vida autónoma e independiente que suele ser, en nuestro contexto sociocultural y a pesar de retrasarse cada vez más, la llamada edad de emancipación, la propia de la edad adulta, es decir, la del grupo de edad en la que se sitúan los enfermos crónicos de esta investigación. Pero ¿cómo se hace para poder vivir solo cuando no se puede por limitaciones de salud? Las políticas públicas no dan respuesta a ello puesto que se da por sentado que la familia o red social es la principal responsable de ocuparse, de allí que, como ya fue mencionado, entre el $75 \%$ y el $88 \%$ de los cuidados de larga duración los realice la red social del enfermo ${ }^{(9,10,11)}$. Pero además, el modelo diseñado de atención a la dependencia en España basado en una lógica funcional del cuerpo no tiene en cuenta las necesidades y voluntades individuales.

Como contrapunto, el modelo propuesto por el movimiento de vida independiente da cuenta de diversas soluciones que no solo mejoran la calidad de vida de las personas y les permite llevar a cabo su vida de forma (más) independiente, sino que además ha demostrado que disminuye los costos para el Estado ${ }^{(18,19)}$. Este movimiento social nació en el marco de la lucha por los derechos civiles de finales de los años sesenta en EE.UU., impulsado por personas con diversidad funcional que necesitaban asistencia personal para llevar a cabo sus actividades diarias. Su objetivo era que las personas discriminadas por su diversidad funcional pudieran salir de los hospitales e instituciones, e incluso de sus casas, en donde se hallaban recluidas, para que pudieran vivir y participar en la comunidad $^{(20,21)}$. Cabe decir que sus propuestas están principalmente orientadas a las diversidades funcionales $y$, por tanto, presentan diferencias respecto a las situaciones de algunos enfermos crónicos. Sin embargo, sus propuestas y soluciones serían un buen punto de partida a tener en cuenta para tratar de contribuir, en la medida de lo posible, a crear un modelo social y político que dé respuesta a las necesidades reales de los enfermos crónicos, por ejemplo, incorporando esas propuestas e ideas al Programa de Atención Individualizado (PAI) de atención sociosanitaria que existe en nuestro país, pero que 
demuestra serias limitaciones en la práctica. La necesidad de contar con un nuevo modelo no solo beneficiaría a los que quieren seguir viviendo solos de forma independiente (los singles) sino al conjunto de adultos jóvenes con enfermedades crónicas.

\section{En pareja}

La segunda variante es la de los que viven en pareja. Si a priori uno podría pensar que la situación es radicalmente diferente a la de los singles, los datos empíricos demuestran que esto no siempre es así. Presuponer de entrada que los enfermos crónicos que viven en pareja tienen un apoyo principal asegurado que cuidará de ellos es un error. Tienen ayuda sí, pero la ayuda no siempre llega hasta donde ambos quisieran. Por lo general, las parejas de los enfermos crónicos deben trabajar, sobre todo si el enfermo no trabaja, puesto que alguien tiene que hacer frente a los gastos y, en la mayoría de los casos, trabajan fuera de casa. Este tipo de trabajo impone un calendario de los tiempos familiares difícil de compaginar con los cuidados al familiar enfermo $^{(22,23)}$. Tal como ocurre con la actual problemática de conciliación de la vida familiar y laboral en España, centrada en el cuidado de los hijos, la conciliación resulta difícil o imposible en el caso de parejas de mediana edad en la que un miembro debe trabajar y el otro está enfermo/a en casa. En este sentido, y paradójicamente, el enfermo crónico vive una situación parecida a como si viviera solo; aquellos que no trabajan, que están de baja temporal o tienen una discapacidad permanente, pueden Ilegar a pasar muchas horas solos en casa cuando la pareja se va a trabajar. Y mientras están solos, no hay nadie a quien acudir en caso de necesidad, como le ocurrió a Paco:

Estaba muerto de sed y no me podía levantar de la cama... y estábamos a 40 grados... y tuve que esperar a que mi mujer volviera del trabajo para poder beber un vaso de agua... (Paco, 32 años)
Otro aspecto a tener en cuenta con respecto a la pareja es el momento de la relación en el que la enfermedad irrumpe: no es lo mismo en una pareja solida con años de relación, que si la relación es más reciente. En general, la pareja sufre una disrupción biográfica y relacional importante cuando la enfermedad irrumpe en sus vidas ${ }^{(15,24,25)}$. En algunos casos, se generan conflictos a raíz de las dificultades que entraña la nueva realidad de convivir tanto con la enfermedad como con el enfermo, dos entidades que pueden entenderse por separado. Una cosa son las necesidades (objetivas) que genere la enfermedad y otra cosa las necesidades (subjetivas) que el propio enfermo considere que tiene. La concordancia o disonancia entre ambas juega un papel relevante en la aceptación, adaptación y gestión de la enfermedad por parte de los miembros de la pareja y afectará cómo se entenderán y llevarán a cabo las prácticas de cuidado y apoyo.

La bibliografía sobre cronicidad suele poner la mirada en los problemas logísticos del día-a-día, en el cuidado del enfermo crónico como secuencia y suma de actividades diarias y caseras $^{(26)}$, en los procesos de adaptación y acomodación por parte de ambos miembros a la nueva situación y en relación con los cambios en el estilo de $\operatorname{vida}^{(27,28,29,30)}$ y en la carga física y emocional del cuidado prolongado en el tiempo ${ }^{(31)}$. Sin embargo, a pesar de algunas pocas publicaciones, aún faltan estudios que profundicen en las consecuencias que los conflictos cotidianos y el desgaste de convivir con la enfermedad crónica puede conllevar para la pareja, pudiendo acabar en separación o divorcio $^{(15,32,33,34,35,36)}$. Los enfermos crónicos -sobre todo las mujeres que participaron en esta investigación- son muy conscientes de ello, y creen que "se habla poco de ello". Esto se podría explicar en parte porque, en el contexto sociocultural de esta investigación, dejar a la pareja cuando está enferma no está bien visto. No podemos adentrarnos aquí en el complejo análisis de cómo la enfermedad crónica afecta la vida de pareja porque "cada casa es un mundo" como se dice popularmente en España, pero para el tema que nos 
ocupa, se pone de relieve que, tanto en el caso que la pareja no pueda dar respuesta a las necesidades del otro miembro enfermo, como si la relación termina, esto conlleva la ausencia significativa de apoyo informal continuado para el enfermo crónico adulto.

\section{Los hijos}

Cuando en una pareja joven uno de los dos padece de una enfermedad crónica con ciertas afectaciones para la vida diaria, la situación se complica si tienen hijos, dado que estos también requieren atenciones y alguien debe hacerse cargo. Adonde no llegue el miembro de la pareja enfermo deberá llegar el otro y, en ocasiones, esto puede conllevar a que toda la responsabilidad familiar del día-a-día recaiga en un solo individuo, como relata Joan respeto de su pareja:

\begin{abstract}
...la dependencia, o sea claro, de yo conducir [...] Me encontraba que la mujer tenía que asumir muchas cosas que yo hacía [...] La dependencia que tenía de los demás. Ella conducía, ella llevaba el niño al cole, ella se ocupaba de la casa, los papeles, de la enfermedad... porque claro yo no podía. Y además, trabajando, porque ella trabaja, claro. (Joan, 49 años)
\end{abstract}

Muchas de estas parejas de mediana edad tienen hijos pequeños o adolescentes. Para ellos, tener un progenitor enfermo o tener que cuidar de su progenitor enfermo no es algo que se espere como habitual y no está exento de impacto emocional. Sin embargo, según lo observado en esta investigación -y con toda la provisionalidad de esta muestra micro- parecería como si los progenitores llevaran peor la carga de la enfermedad crónica que sus hijos, quienes parecían Ilevarla mejor. Algunos progenitores expresan la frustración que experimentan por no poder ocuparse de los hijos como quisieran y tienen la sensación de perderse la vida de los hijos, ya que quizás no pueden disfrutar de actividades conjuntas como quisieran: "yo me saltaría a jugar al fútbol con ellos pero no puedo" se lamentaba Rafa, "siempre tirada en la cama ¿qué pensaran?, iqué mala madre!" decía Montse. Con ello, se sienten culpables tanto por no poder atender a los hijos como por la carga que la enfermedad suponga para sus hijos: "no quiero que sufran por lo mío", decía María.

Para otras parejas jóvenes, que desean tener hijos, la situación se puede complicar a raíz de la enfermedad, porque son conscientes de las limitaciones de uno de los miembros de la pareja. Otros no podrán tener hijos a pesar de querer tenerlos porque los médicos lo han desaconsejado a raíz de la enfermedad que padecen. Otros, no se verán con fuerzas para asumir dicha responsabilidad, como el caso de Isabel:

\begin{abstract}
¿Cómo debo tener yo un crío? Yo a las diez [22:00] ya estoy en la cama. ¿Y si se me despierta cada hora?... Anda, anda, anda. Pánico me da. Yo necesito dormir muchas horas, si no, no tengo pilas. Tengo miedo de volver a caer en el agujero negro [crisis en la que se encontró muy mal]. Y el marido también tiene miedo: "¿y si me vuelves a caer Isabel?", me dice. ¿Cómo tengo que tener el crio yo? ¿Y después qué? ¿Todo pa él? Quita, quita. (Isabel, 36 años)
\end{abstract}

A las limitaciones físicas que la enfermedad impone en un cuerpo aún joven, se añaden las limitaciones para asumir los roles, en cuanto a cumplir con las responsabilidades y funciones sociales que, por ejemplo, como progenitor o como pareja se espera de ellos $^{(34,37,38,39)}$. Esto genera conflictos en el ámbito doméstico e incomprensión tanto en el ámbito familiar como social. La situación de Montse es un claro ejemplo de esta problemática:

Estoy todo el día en casa y no puedo ni siquiera con las tareas de casa, y mi marido no lo entiende, ni mi situación ni mi enfermedad: "¿qué estás haciendo todo el día aquí?" me dice. Discutimos... un día se irá... ya ni nos besamos... mis hijos adolescentes necesitan a su madre 
y mamá está tirada en la cama inmersa de dolor, y no entienden por qué no me levanto de la cama a las nueve de la noche para prepararles la cena... y mis padres se están haciendo mayores y me necesitan más, me necesitan a mí... y yo... no puedo, no puedo, pero no lo entienden. (Montse, 50 años)

\section{Los progenitores}

Algunos enfermos crónicos adultos viven con sus progenitores. Esto fue un hallazgo sorprendente que esta investigación puso de relieve y que no se acostumbra a reflejar en la bibliografía sobre el tema. Esto significa que nos encontramos con personas de treinta o cuarenta años y más, que viven y dependen de los cuidados de unos progenitores ya mayores de sesenta, setenta o más años. Las casuísticas que llevan a esta situación son principalmente dos: 1) personas que aún vivían con los progenitores y a las que la irrupción de la enfermedad los limitó en un grado que les impidió irse e independizarse; 2) personas que ya se habían emancipado (y vivían solos o en pareja) pero que tuvieron que volver a casa de los progenitores debido a la imposibilidad de vivir solos a raíz de la aparición de la enfermedad, a su empeoramiento, o a raíz de una separación o divorcio. Cuando hablamos de progenitores, esto puede implicar volver al hogar familiar con ambos progenitores, o ir a vivir con uno de ellos (padre o madre) en el caso de que estos se hubiesen separado, o uno de ellos faltara. Sea como sea, depender de los cuidados de sus progenitores de avanzada edad (y no al revés) entraña una situación individual compleja de pérdida de autonomía e independencia y puede conllevar, en algunos casos, un proceso de infantilización del adulto enfermo dependiente que no puede vivir su vida con la autonomía, independencia, libertad, privacidad e intimidad que quizá desearía (salvando las excepciones en las que eso sea realizable a pesar de todo).

Por otro lado, la carga y desazón que esto supone para unos progenitores mayores es notoria, no solo por la carga física y emocional del cuidado del hijo adulto enfermo, sino también en cuanto a la posibilidad de que los progenitores enfermen y requieran de cuidados que los hijos no pueden proporcionar. El peor de los escenarios, que ni unos ni otros quieren imaginar, es la muerte del padre o de la madre que cuida del hijo adulto enfermo. Mariona, que a sus 36 años vivía con su madre de 66 , que era su principal cuidadora, se preguntaba: "¿qué será de mí cuando mi madre no esté?, ¿quién cuidará de mí?". Su madre murió hace dos años. El novio de Mariona pasó a ocupar el rol de cuidador principal. Desde entonces viven juntos, son una pareja fuerte que ha pasado por situaciones críticas y ha sobrevivido y piensan casarse.

\section{El curso cíclico de la enfermedad crónica}

El curso cíclico de la enfermedad crónica determina y condiciona las necesidades de atención que el enfermo precisa en mayor o menor medida a lo largo del tiempo. Independientemente de la gran variabilidad de padeceres crónicos respecto al nivel de limitaciones, discapacidad o dependencia que presentan, lo que tienen en común es que la mayoría cursa por brotes cíclicos de fases estables seguidas de crisis o reagudización de síntomas, para volver a la fase estable, y así a lo largo de los meses y años, lo que los enfermos denominan "temporadas buenas" o "temporadas malas".

El curso y evolución de la enfermedad no siempre cumple con un curso natural preestablecido como la biomedicina ha tendido a presentar, sino que puede ser azarosamente caprichoso. Durante las temporadas buenas, el enfermo lleva a cabo sus regímenes diarios de autocuidado y precisa de ayuda puntual según sea su situación, sus limitaciones o grado dependencia. En cambio, durante las temporadas malas, puede precisar de mayor ayuda puntual, ya que suelen ir acompañadas de mayor malestar, dolor, sufrimiento, discapacidad, lo que afecta la autonomía del 
individuo y lo convierte temporalmente en una persona más dependiente $\mathrm{y}$, por tanto, limita su propia respuesta de autocuidado. Es durante las malas temporadas cuando se hace especialmente necesario el apoyo informal y se ponen en evidencia las limitaciones de ese apoyo.

No hay que entender que durante las temporadas buenas todo funciona bien y que el enfermo no necesita ningún tipo de ayuda, también hay días buenos y días malos ${ }^{(15)}$. Si bien hay enfermedades crónicas que son más fácilmente autogestionables, en otros casos, esos ciclos buenos o malos se reducen a semanas, días e incluso horas de bonanza o todo lo contrario, sin que ello sea previsible. Como bien expone Clara:

Ahora estás bien, ahora no lo estás. Por la mañana te levantas bien y al mediodía estás que no te aguantas y te tienes que poner en la cama. Y luego se te pasa y ya vuelves a funcionar. Es muy difícil de entender. Va así, ad libitum. Un día estás bien, y el otro parece como si te hubiera pasado un camión por encima. Tres días bien y dos fatal, y no sabes ni tú porqué. No hay patrón. Nunca sabes muy bien, tienes que ir arreglándotelas como puedes. Cuando tienes una temporada buena es fantástico, pero cuando notas que viene una mala, uf, da miedo, porque no sabes lo que va a durar, y sabes que lo pasarás mal. (Clara, 36 años)

Estos vaivenes en el estado general de salud del enfermo crónico tienen diversas implicaciones para los ámbitos familiar, social y laboral, que presentan dificultades para comprender por qué ahora sí y por qué ahora no, por qué ayer sí y hoy no, la semana pasada sí y la siguiente no, la persona puede hacer (o no) tal cosa o tal otra. La desatención de los roles familiares y sociales, y el incumplimiento ocasional-continuado de las responsabilidades y funciones sociales y laborales puede generar malos entendidos y conflictos en todos los ámbitos ${ }^{(17,34,35,36,37,38,39,40)}$. De nuevo, en estas fluctuantes fracciones de tiempo -días, horas, semanas- bien dentro de una temporada buena o mala, es cuando el papel de los cuidados informales toma especial relevancia para proveer, en la medida de lo posible, el mayor apoyo requerido. El problema reside en si la red informal de apoyo puede asumir o compaginar unas necesidades mayores de atención, que a pesar de ser puntuales en el tiempo, son puntualmente-continuadas en el tiempo. Esto requiere de un esfuerzo y ajustes personales para los cuidadores o red de apoyo no siempre fáciles de conciliar con otras responsabilidades existentes, lo que evidencia, una vez más, las limitaciones e insostenibilidad del modelo informal de cuidados.

\section{La respuesta social a la enfermedad}

Las relaciones interpersonales entre el enfermo y el familiar, amigo o vecino incidirán significativamente en el dispositivo informal de cuidado a lo largo del tiempo ${ }^{(15,17,36)}$. Cuidar a una persona puntualmente, en forma diaria, durante semanas, meses o años o para siempre, no es lo mismo. Cuidar cansa física, emocional y mentalmente, más para quien asume el rol de cuidador principal, pudiendo llegar a "quemarse" (burn out) o claudicar ${ }^{(31,41)}$. Lo que los propios participantes reconocen también es que "las quejas cansan, para los que tienen que aguantarnos", decía Montse. El estado de ánimo de algunas personas con enfermedades crónicas puede verse afectado por los malos días o las malas temporadas. Laura explicaba cómo "el dolor agria el carácter, y eso hace que tengas mala leche, y claro... estás insoportable". Esto no lo hace fácil para los que están en el entorno cercano del enfermo.

Por otro lado, la dimensión temporal de los cuidados informales de larga duración explica en parte sus limitaciones: el cuidado ilimitado en el tiempo, al que no se le ve fin, tiene un impacto físico y emocional notable para el cuidador principal. Por ello, se hace indispensable que, desde el Estado, se provean mecanismos de apoyo y programas oficiales de respiro al cuidador informal principal; que existen, pero no cubren las necesidades de todos los afectados. Pero también aquí resulta 
especialmente relevante la importancia de la red social de apoyo más amplia del enfermo crónico, para que la carga no resida solo en una persona. Los otros miembros de la familia, amigos y los indispensables vecinos juegan un papel primordial de apoyo informal, tanto a la persona enferma como a su cuidador informal principal. Sin embargo, "tener una amplia red social (familia y amigos) no garantiza por sí mismo que se va a contar con apoyo suficiente cuando sobreviene una necesidad de ayuda de carácter prolongado o permanente ${ }^{\prime \prime(41)}$. Algunas personas de esta red social, sin embargo, no son conscientes de la importancia de su apoyo para el familiar, vecino o amigo enfermo y para su cuidador principal. En ocasiones, la gente desconoce lo que puede hacer, no saben qué pueden aportar ellos o cómo pueden ayudar. Esto tiene mucho que ver en cómo entendemos las prácticas de cuidados informales, qué entendemos por cuidados y qué entendemos por cuidar, pero también tiene que ver con la comunicación entre unos y otros.

Por un lado, se tiende a pensar en prácticas de cuidado bajo un modelo biomédico que distingue principalmente dos niveles de atención: el sanitario y el sociosanitario, cuyas prácticas asistenciales se orientan sobre todo hacia la cura o el cuidado de los aspectos sanitarios, como higiene personal, alimentación, curas, administración medicación, y logísticos, como tareas de la casa, comprar, cocinar, ayuda en los desplazamientos. Por otro lado, se tiende a pensar en cuidados informales como meras tareas funcionales o actividades a realizar. Si bien es cierto que el apoyo al enfermo crónico tiene que ver con esas tareas concretas que desempeñan personas concretas -la pareja que se ocupa de las tareas de la casa, el vecino que compra el pan, el amigo que acompaña a la visita del médico- se aboga aquí por una mirada que tenga en cuenta la actitud y el trato en la interacción entre la persona enferma y su red social de apoyo (cuidadores o no), rescatando la necesidad de atender a los aspectos morales, emocionales y sociales que muchas de las enfermedades crónicas conllevan. Hace unas décadas, en España, se iba a la casa de los enfermos a "hacerles compañía", a charlar, a escuchar la radio, a jugar a las cartas o distraerles, en definitiva, aportar calor humano y compartir (a ratos) la experiencia de la enfermedad. Eso también es cuidar. Ahora raramente se visita a los enfermos en sus casa para "hacerles compañía", a no ser que se trate de una enfermedad degenerativa o fatal, o su vida esté en riesgo o acabándose. A veces, ni se los Ilama por teléfono para saber cómo están. En este contexto, se hace necesario rescatar a un clásico que nos abre la puerta a repensar los cuidados y el cuidar, según Illich ${ }^{(42)}$ :

La asistencia más tradicional a la salud era un programa para dormir, comer, amar, trabajar, jugar, soñar, cantar y sufrir [...] y la curación era una forma tradicional de consolar, cuidar, reconfortar, aceptar, tolerar y también de rechazar al afligido. $^{(42)}$

Otro aspecto importante a tener en cuenta es la carga moral del cuidado: ipoder o querer cuidar? La responsabilidad de proporcionar este tipo de ayuda, atribuida al familiar o al amigo, conlleva una notable carga moral en la sociedad española, tradicionalmente moralista, y genera sentimientos ambivalentes. Aun queriendo ayudar a sus seres queridos:

\footnotetext{
El sentido de obligación moral que todavía impregna a la sociedad española con respecto a los cuidados de larga duración provoca emociones positivas en la relación de ayuda y, al mismo tiempo, sentimientos encontrados ante la dificultad de conciliarlos con la vida personal y el trabajo. La ambivalencia emocional de las personas cuidadoras es una de sus características fundamentales. ${ }^{(41)}$.
}

Los procesos de enfermedad son una experiencia social y un fenómeno cultural, y la respuesta social al enfermo es un acto moral $^{(15,28,35,36,43)}$. Los individuos viven en un mundo social y moral de compromisos. Por ello, las necesidades crónicas de los enfermos 
y las demandas para sus familiares o amigos, deben estar en concordancia con la naturaleza personal de cada relación ${ }^{(15,17,36)}$. Cuidar a los familiares, amigos o vecinos enfermos es un compromiso que se vincula con el concepto de reciprocidad ${ }^{(44)}$ cuyas reglas sociales y acuerdos implícitos o explícitos se fundan en un actualizado modelo de comunidad, el escenario en el que se llevan a cabo estas prácticas.

Curiosamente, los participantes explican la respuesta de su red social de apoyo en términos de buena o mala suerte. Algunos agradecen la suerte que han tenido: "yo he tenido mucha suerte con mi familia y amigos, que siempre han estado ahí y me han ayudado mucho", "mi marido es un diez, no puedo pedir más", mientras que otros lamentan las carencias y la falta de apoyo. Lo significativo de entender la ayuda recibida en términos de suerte pone de relieve dos cosas: la primera, es que existen presunciones sobre lo que los demás hacen o se cree que deberían hacer y, la segunda, que estas valoraciones generalmente tienen que ver más con las actitudes y el trato recibido por parte de los familiares y allegados, es decir, con la calidad percibida de la ayuda.

Lamentablemente, esta investigación ha encontrado más casos de mala suerte en la respuesta social a la enfermedad y en la calidad del cuidado informal recibido. Varios participantes han referido, por ejemplo, que sus parejas se molestaban por tener que llevarles un vaso de agua a la cama o que no querían ir a pasear con ellos porque "andan lentos". Todos han referido haber experimentado mucha incomprensión: "la gente no lo entiende", "la gente no lo comprende" son narrativas habituales. La mayoría ha relatado la ausencia y paulatina desaparición de familiares o amigos bajo el pretexto de "no quiero molestar" o "ya sabemos que si necesitas algo, nos Ilamarás". Estos problemas tienen que ver tanto con cómo se percibe y valora socialmente el tipo de enfermedad que la persona padezca, como con los patrones culturales y expectativas sociales atribuidos a este grupo concreto de edad de adultos jóvenes $^{(7,45)}$. Todo ello incide significativamente en la respuesta social a la enfermedad y al enfermo, es decir, a poner en marcha una red social de cuidado informal adecuado, que debe ser analizada atendiendo a la diversidad de situaciones y circunstancias personales, y sobre la base del tipo de relación interpersonal entre la persona y su familiar o amigo. En este sentido "cada relación es un mundo" y no podemos generalizar. Sin embargo, las carencias relatadas nos avisan de aquello que no va bien y es susceptible de mejorar, poniendo en evidencia, nuevamente, las limitaciones de los cuidados informales de larga duración.

\section{CONCLUSIONES Y PROPUESTAS}

Respecto a las políticas públicas, el escenario de la atención a las enfermedades crónicas ha cambiado sustancialmente en la última década desde que los expertos de la OMS advirtieron en 2003 el nuevo reto ${ }^{(46)} \mathrm{e}$ instaron a los países europeos a desarrollar e implementar planes nacionales de atención a la cronicidad ${ }^{(1,47,48)}$. En España, el primer intento fue con la Ley 39/2006 denominada Ley de Dependencia ${ }^{(8)}$, insuficiente en la práctica tanto por motivos de diseño como por problemas de financiación, que puso en evidencia sus limitaciones ante la amplia diversidad y casuística de las enfermedades crónicas y excluyó a un notable número de personas con enfermedades crónicas que no pueden obtener ni las prestaciones ni los beneficios que esta ley dispone ${ }^{(49)}$. Recientemente, en 2011 y 2012, se han diseñado planes y programas específicos de atención a las enfermedades crónicas en el estado español ${ }^{(4,5)}$. Sin embargo, aún no se puede hablar de implementación efectiva o que se esté dando respuesta a las necesidades sociosanitarias del conjunto de afectados. Pero ¿qué Ilama la atención en esos planes, programas y estrategias de abordaje a la cronicidad? Que, en gran medida, se sigue contando con la red de cuidados informales y se confía en la autoatención como solución al empeoramiento de la situación. En este contexto, cabe 
preguntarse: si volvieran a hacer los estudios que hace poco más de una década cifraron en torno al $80 \%$ los cuidados informales para los enfermos crónicos ¿cuál sería la cifra ahora?, ¿cuánto habrá cambiado gracias a la Ley de Dependencia y a los incipientes programas de atención a la cronicidad?

Aun sin tener la cifra actualizada, no cabe duda de que la responsabilidad de los cuidados de larga duración en España se reparte de forma totalmente desequilibrada entre los servicios que ofrece el Estado y las estrategias de cuidado informal que pone en funcionamiento la persona enferma y su entorno cercano de apoyo. La mirada desde la antropología médica permite mostrar la distancia existente entre los modelos que proponen los expertos profesionales (outsiders) y las necesidades reales de los expertos enfermos (insiders). La solución pasa por una mirada interdisciplinar y un trabajo colaborativo entre las ciencias sociales, la biomedicina y los que diseñan las políticas públicas. El reto es el de construir entre todos un modelo de atención a la cronicidad que dé respuesta a la gran variedad de casuísticas y circunstancias personales y sociales de la enfermedad. Eso pasa, inexorablemente, por escuchar la voz de los afectados, y por la necesaria articulación entre la autoatención y el cuidado informal y la gestión por parte de las políticas públicas.

\section{AGRADECIMIENTOS}

Este artículo expone resultados parciales del material etnográfico recogido durante mi investigación doctoral titulada "El temps del mal. L'experiència i la gestió de la cronicitat en adults" [El tiempo del mal. La experiencia y la gestión de la cronicidad en adultos]. Dicha investigación doctoral fue posible gracias a una beca predoctoral de la Agència de Gestió d'Ajuts Universitaris i de Recerca para personal investigador novel (AGAUR-FI), con el apoyo del Comisionado para Universidades e Investigación del Departamento de Innovación, Universidades y Empresa de la Generalitat de Catalunya (España) y del Fondo Social Europeo (Números de Expedientes 2009FI_B 00115; 2010FI_B1 00104; 2011FI_B2 00101), además de una bēca de movilidad con el apoyo de la Secretaria de Universidades e Investigación y del Departamento de Economía y Conocimiento de la Generalitat de Catalunya (España) (Número de Expediente: 2011 BE1 00518).

\section{REFERENCIAS BIBLIOGRAFICAS}

1. Organización Mundial de la Salud. Preparación de los profesionales de la atención de salud para el siglo XXI: El reto de las enfermedades crónicas. Ginebra: Ediciones de la OMS; 2005.

2. Organización Mundial de la Salud. Estrategia mundial sobre régimen alimentario, actividad física y salud marco para el seguimiento y evaluación de la aplicación. Ginebra: Ediciones de la OMS; 2006.
3. Instituto Nacional de Estadística. Encuesta Europea de Salud en España. Madrid: INE; 2014.

4. Ministerio de Sanidad, Servicios Sociales e Igualdad. Estrategia para el abordaje de la cronicidad en el Sistema Nacional de Salud. Madrid: Ministerio de Sanidad, Servicios Sociales e Igualdad; 2012.

5. Programa de Prevenció i Atenció a la Cronicitat. Dossier de presentació. Barcelona: Departament de Salut, Generalitat de Catalunya; 2011.

6. García F, Sarriá A, Hernández A, Alcázar Raimundo. Revisión de intervenciones con nuevas tecnologías en el control de las enfermedades crónicas. Madrid: AETS-ISCIII; 2005.

7. Masana L. El temps del mal. L'experiència i la gestió de la cronicitat en adults [Internet]. 2013 [Tesis de doctorado]. Tarragona: Departamento de Antropología, Filosofía y Trabajo Social, Universitat Rovira i Virgili [citado 10 oct 2016]. Disponible en: http://www.tdx.cat/handle/10803/119547.

8. España, Congreso de los Diputados. Ley 39/2006: Ley de Promoción de la Autonomía Personal y Atención a las Personas en Situación de Dependencia. Madrid: Ministerio de Trabajo y Asuntos Sociales; 2006.

9. García-Calvente MM, Mateo-Rodríguez I, Gutiérrez, P. Cuidados y cuidadores en el sistema informal de salud. Granada: Escuela Andaluza de Salud Pública; 1999.

10. Durán MA. Las demandas sanitarias de las familias. Gaceta Sanitaria. 2004;18(Supl 1):195-200. 
11. Bofill S. Cuidado formal e informal en Cataluña: Un análisis de la percepción de las mujeres mayores en relación a sus derechos y obligaciones en materia de cuidados y atención. Etnográfica. 2006;10(2):263-281.

12. Haro JA. Cuidados profanos: una dimensión ambigua en la atención de la salud. En: Comelles JM, Perdiguero E, (eds.). Medicina y cultura: Estudios entre la antropología y la medicina. Barcelona: Bellaterra; 2000.

13. Comelles JM. De la ayuda mutua y la asistencia como categorías antropológicas: Una revisión conceptual. Trabajo Social y Salud. 2000;(35):151-172.

14. Menéndez E. Modelos de atención de los padecimientos: de exclusiones teóricas y articulaciones prácticas. Ciencia \& Saúde Colectiva. 2003;8(1):185-207.

15. Charmaz K, Good Days. Bad days: The self in chronic illness and time. New Brunswick: Rutgers University Press; 1991.

16. Goffman E. Relaciones en público: Microestudios del orden público. Madrid: Alianza Editorial; 1979.

17. Goffman E. Estigma: La identidad deteriorada. 10a ed. Buenos Aires: Amorrortu; 2006.

18. Pié Balaguer A, (coord.). Deconstruyendo la dependencia: Propuestas para una vida independiente. Barcelona: Universitat Oberta de Catalunya; 2012.

19. García Alonso JV, (coord.). El movimiento de vida independiente: Experiencias Internacionales. Madrid: Fundación Luis Vives; 2003.

20. Oficina Vida Independent. El movimiento de vida independiente [Internet]. c2017 [citado $10 \mathrm{sep}$ 2016]. Disponible en: http://tinyurl.com/ldxmf6z.

21. Foro de Vida Independiente y Divertad [Internet]. 2016 [citado 10 sep 2016]. Disponible en: http://tinyurl.com/ygv38nw.

22. Aguirre R. Los cuidados familiares como problema público y objeto de políticas. En: Arriagada I, (ed.). Políticas hacia las familias, protección e inclusión sociales. Santiago de Chile: Naciones Unidas; 2005.

23. Durán MA. Los costes invisibles de la enfermedad. Bilbao: Fundación BBVA; 1999.

24. Bury M. Chronic illness as biographical disruption. Sociology of Health \& Illness. 1982;4(2): 167-182.
25. Becker G. Disrupted lives: How people create meaning in a chaotic world. California: University of California Press; 1997.

26. Corbin J, Strauss A. Unending work and care: Managing chronic illness at home. San Francisco: Jossey-Bass; 1988.

27. Radley A. Style, discourse and constraint in adjustment to chronic illness. Sociology of Health \& Illness. 1989;11(3):230-252.

28. Radley A. Worlds of illness: Biographical and cultural perspectives on health and disease. London: Routledge; 1993.

29. Radley A, Green R. Bearing illness: Study of couples where the husband awaits coronary graft surgery. Social Science \& Medicine. 1986;23(6):577-585.

30. Radley A, Green R. Illness as adjustment: a methodology and conceptual framework. Sociology of Health \& Illness. 1987;9(2):179-207.

31. Maslach C. Burnout: the cost of caring. New York: Prentice-Hall Press; 1982.

32. Allué M. DisCapacitados: La reivindicación de la igualdad en la diferencia. Barcelona: Bellaterra; 2003.

33. Strauss A, Corbin J, Fagerhaugh S, Glaser B, Maines D, Suczek B, Wiener CL. Chronic illness and the quality of life. Sant Louis: Mosby; 1984.

34. Devillard MJ, Otegui R, García P. La voz callada: Aproximación antropológico-social al enfermo de artritis reumatoide. Madrid: Comunidad de Madrid, Consejería de Salud; 1991.

35. Frank AW. The wounded storyteller: Body, illness and ethics. Chicago: The University of Chicago Press; 1995.

36. Kleinman A. The illnes narratives: Suffering, healing and the human condition. New York: Basic Books; 1988.

37. Estroff SE. Identity, disability and schizophrenia: The problem of chronicity. In: Lindenbaum S, Lock M, (eds.). Knowledge, power, and practice: The anthropology of medicine and everyday life. Berkeley: University of California Press; 1993.

38. Söderberg S, Strand M, Haapala M, Lundman $B$. Living with a woman with fibromyalgia from the perspective of the husband. Journal of Advanced Nursing. 2006;42(2):143-150. 
39. Ware NC. Toward a model of social course in chronic illness: The example of chronic fatigue syndrome. Culture, Medicine \& Psychiatry. 1999;23:303-331.

40. Miles A, Curran HV, Pearce S, Allan L. Managing constraint: The experience of people with chronic pain. Social Science \& Medicine. 2005;61(2):431-441.

41. Ministerio de Trabajo y Asuntos Sociales. Atención a las personas en situación de dependencia en España: Libro Blanco. Madrid: Ministerio de Trabajo y Asuntos Sociales, IMSERSO; 2004.

42. Illich I. Némesis médica: La expropiación de la salud. Barcelona: Barral Editores; 1975.

43. Kleinman A, Benson P. La vida moral de los que sufren enfermedad y el fracaso existencial de la medicina. Humanitas Monográfico. 2004;2:17-26.

44. Mauss M. Ensayo sobre los dones: Motivo y forma del cambio en las sociedades primitivas. En: Mauss M. Sociología y antropología. 2a ed. Madrid: Tecnos; 1971.
45. Masana L. Invisible chronic illnesses inside apparently healthy bodies. In: Fainzang S, Haxaire C, (eds.). Of bodies and symptoms: Anthropological perspectives on their social and medical treatment. Tarragona: Publicacions URVCol•lecció Antropologia Mèdica; 2011.

46. World Health Organization. Key policy issues in long-term care. Geneva: WHO; 2003.

47. World Health Organization. A strategy to prevent chronic disease in Europe: A focus on Public Health Action, the CINDI Vision. Geneva: WHO; 2004.

48. World Health Organization. Towards a European strategy on noncommunicable diseases. Geneva: WHO; 2004

49. Masana L. Self-Care and management of adults with chronic illness and dependency: The Spanish case in the context of the new dependency law. In: Fainzang S, Einar Hem H, Bech Risor M, (eds.) The taste for knowledge: Medical anthropology facing medical realities. Denmark: Aarhus University Press; 2010. 